\title{
Factors contributing to hypoglycemia among patient with diabetes presenting with symptomatic hypoglycemia to a tertiary care centre of northern Sri Lanka.
}

\author{
Niranjini Perinpanathan ${ }^{1}$, Thirunavukarasu Kumanan ${ }^{2}$, Aravinthan Mahalingam ${ }^{1}$ \\ ${ }^{1}$ Teaching Hospital Jaffna \\ 2University of Jaffna
}

\section{Abstract}

Prevalence of hypoglycemia is in increasing trend among diabetic patients especially when a stringent glycemic control is warranted. This study analyzes both traditional and drug related precipitating factors and the knowledge regarding hypoglycemia among diabetic patients presented with symptomatic hypoglycemia to a tertiary hospital in Northern Sri Lanka. It was a descriptive cross-sectional study. All adult diabetic patients who were admitted with symptomatic hypoglycemia were recruited $(n=62)$. Majority $(93.5 \%)$ of them were having type 2 diabetes mellitus $(n=58)$ and $2 / 3$ had evidence of nephropathy. Majority presented with their very first episode (68\%). Twenty-six patients were on Insulin based regime and 29 were on oral hypoglycaemic drugs which contains a sulphonylurea. Among the 7 patients who were on metformin monotherapy all except one had established nephropathy. It was found that 7 patients with renal impairment who were on gliclazide monotherapy developed hypoglycemia. Apart from the drugs, missed meals (52\%), concurrent illness $(46 \%)$, recent hospital stays (25\%), recent escalation in medication $(20 \%)$ also contributed for the hypoglycemic events in these patients. Even though majority of patients had good knowledge on first aid measures only a fraction of them have attempted it. None of the patients were aware about glucagon home injection. Seventy seven percentage of patients were knowledgeable about the devastating complications of prolonged hypoglycemia while only one third was aware that warning signs can be absent in chronic diabetes patients.

Insulin and sulphonylureas are the most potent drugs to cause hypoglycemia, but metformin also can potentiate it when prescribed to patients with nephropathy. Contrary to popular belief, gliclazide is not always safe in renal impairment and should be used with caution.

Key words: hypoglycemia, diabetes, oral hypoglycaemic agents, risk factors, renal impairment, knowledge

Received: $10^{\text {th }}$ March $2018 \quad$ Accepted: 25 $5^{\text {th }}$ June 2018 Published: $31^{\text {st }}$ Aug 2018

Correspondence email: niranji07@yahoo.co.uk

https://orcid.org/0000-0002-3806-3571

This is an open-access article distributed under the terms of the Creative Commons Attribution License, which permits unrestricted use, distribution, and reproduction in any medium, provided the original author and source are credited (CC BY 4.0) 


\section{Introduction}

Strict glycemic control plays a pivotal role in preventing complications among patients with diabetes mellitus at the expense of increasing risk of hypoglycemia. The American Diabetes Association defines hypoglycemia as, plasma glucose level less than $70 \mathrm{mg} / \mathrm{dl}$ accompanied by typical neuroglycopenic and/or sympathetic symptoms, requiring carbohydrate or glucose ingestion, while severe hypoglycemia is defined as low blood sugar requiring the assistance of another person (1). Most of the type 2 diabetic patients require multiple oral hypoglycemic drugs and a significant proportion needs insulin-based regime to achieve optimum glycemic control. Therefore, it is not unusual to see increasing number of patients with hypoglycemic events necessitating emergency admissions. Unfortunately, there are no documented data on prevalence of such events in Sri Lankan population, and the morbidity is often underestimated while the devastating consequences are overlooked.

Our study is designed to find out the common risk factors associated with hypoglycemic events among the patients and also the knowledge on warning symptoms, emergency management and complications of hypoglycemia. Further, this study analyses whether the duration of diabetes, duration of insulin use, degree of renal impairment and association with different classes and types of oral anti diabetic drugs on precipitating hypoglycemic events.

In the past, several landmark studies have analyzed the hypoglycemic events and concluded that frequency of major hypoglycemic events are significantly higher in the intensive glycemic control group compared to less stringent glycemic control group (2). Further, they found that patients who suffered hypoglycemic events have been treated with either insulin or a sulphonylurea (3). The relationship between the duration of insulin use and the duration of diabetes with the frequency of hypoglycemia showed that prevalence is increased with duration of insulin therapy, diabetes and also it is inversely proportionate to HbA1C level (4).

In Sri Lanka, a study which analyzed the knowledge and awareness of hypoglycemia among diabetic patients who were randomly selected at a medical clinic showed that only $42 \%$ of patients were having adequate knowledge about symptoms of hypoglycemia and only $57.6 \%$ were aware that how to manage a hypoglycemic episode (5).

\section{Methods}

This was a descriptive cross-sectional study carried out in emergency department of teaching hospital of Jaffna over a period of 6 months. All adult diabetic patients who were admitted with documented hypoglycemia were recruited for the study excluding the patients with chronic cognitive dysfunction. Details regarding the current admission, and information on diabetes such as type, duration, and current treatment regime, other comorbidities, and the possible precipitating factors were collected from the eligible patients using an interviewer administered questionnaire and also from bed head tickets and clinic books. Further, the knowledge of patients on the immediate management and complications of hypoglycemia was assessed by 8 questions with true / false response. A capillary random blood sugar was checked and recorded on admission and other investigations were performed based on individual patient's clinical presentation. SPSS (version 21) analytical package was used to analyze the data and results are presented as counts and percentages and as mean +/_ SD for continuous variables. A chi square test was used to analyze the results statistically. An approval from Ethical Review Committee of University of Jaffna was obtained prior to the commencement of study and all participants of this study gave their informed written consent.

\section{Results}

A total of sixty-two patients $(n=62)$ have got admitted with hypoglycemia during the study period, and $93.5 \%$ of them were having type 2 diabetes mellitus $(\mathrm{n}=58)$. The incidence was slightly higher in females (58\%). Majority of the patients fall within the age group of 41 - 60 years $(61.3 \%)$. Majority of patients $(67.7 \%)$ presented with their very first episode of hypoglycemic event and rest of them had 2 or more hospital admissions related to hypoglycemia.

The duration of diabetes in this study population varies from less than one year to more than 20 years. About $42 \%$ of patients $(n=26)$ were taking premixed insulin in variable doses with or without oral hypoglycemic agents. Majority of patients were taking a dose in the range of 21-60 units per day.

In this study $2 / 3$ of patients (42 out of 62 ) had evidence of nephropathy on presentation which ranges from positive urine microalbumin to overt renal failure. Thirty-five patients $(56.5 \%)$ had estimated glomerular filtration rate(eGFR) of less than $60 \mathrm{ml} / \mathrm{min}$ and among them 10 patients had end stage renal disease with an eGFR less than $15 \mathrm{ml} / \mathrm{min}$ and was on regular renal replacement therapy.

Regarding the risk factors, majority of the study population 32 out of 62 patients $(52 \%)$ gave a history of a missed meal as a cause for the hypoglycemic episode. And the second common factor was concurrent illness predominantly a respiratory or urinary infection and it was found in almost half of the patient $(n=29)$. Around one third of patients claim that their eating habits were erratic. Unusual physical activity has precipitated the hypoglycemia in $10 \%$ of patients. One fourth of patients had an inward hospital stay 
within a month preceding the event. Twenty percent of patients had escalation of either insulin or oral hypoglycemic drugs during the last outpatient clinic visit or during the recent hospital admission. In our study only 2 participants related alcohol intake to hypoglycemic event. In 10 patients $(16 \%)$ a recent deterioration of renal function has been noted.

Eighty-seven percentage of patients (54 out of 62) who got admitted with hypoglycemia in this study, were well aware of the fact that sugary drink or substance should be taken immediately with the onset of warning symptoms but only $37 \%$ of the study group have received first aid at home. Forty-two percentage of patients (26 out of 62 ) had the knowledge that it should not be attempted in an unconscious patient. None of the patient was aware of home glucagon injection as an immediate first aid treatment for hypoglycemia.

Regarding the awareness on complications of untreated hypoglycemia, 48 out of 62 patients $(77.4 \%)$ were aware that prolonged hypoglycemia can cause permanent brain damage and a similar percentage was aware that it could be fatal if left untreated. Only one fourth of patients (15 out of 62) had the knowledge that hypoglycemia can precipitate acute coronary event or cerebrovascular event. It was important to note that more than half of the patients $(58 \%)$ were not aware that warning symptoms could be absent or subtle, in patients who had diabetes for long duration.

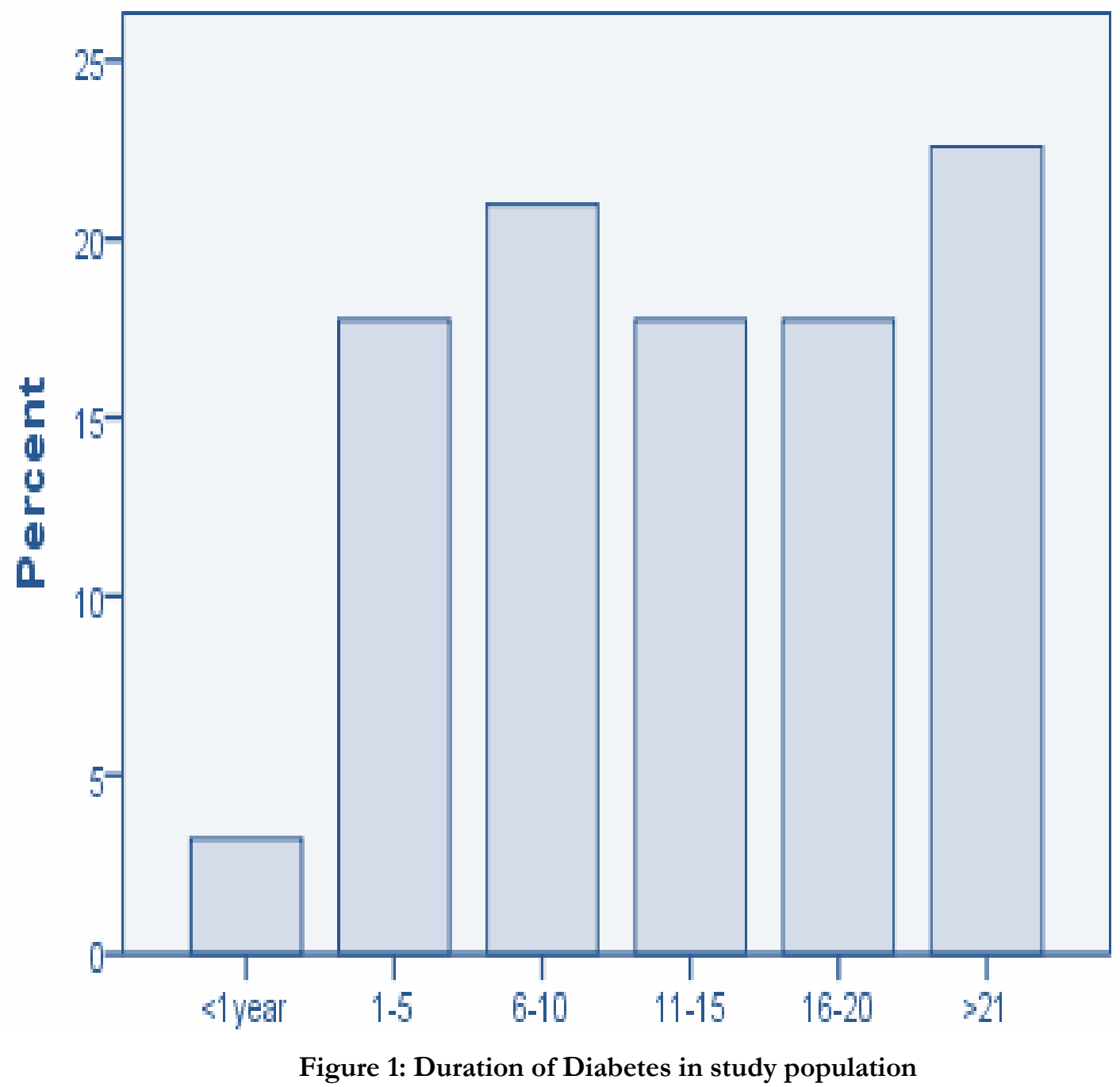




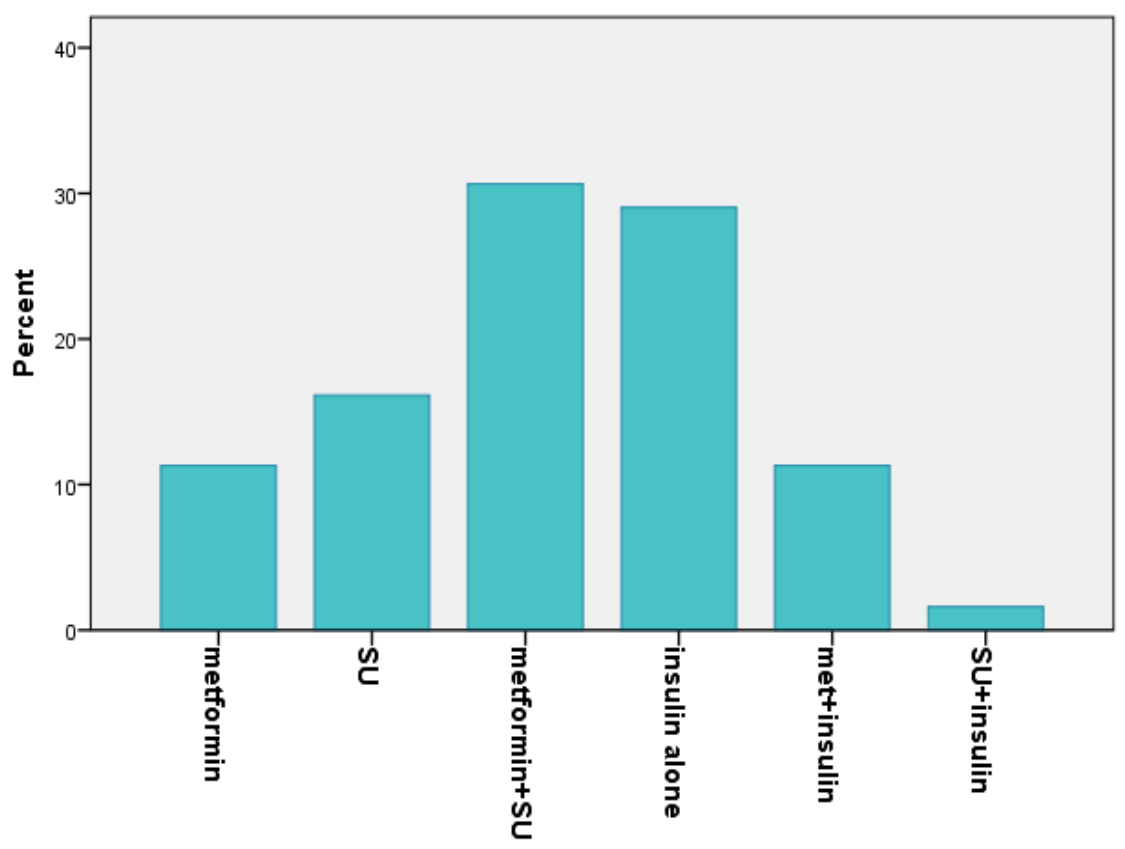

Figure 2: Pattern of drug regimes in the study group

$\begin{array}{ll}\text { Metformin alone } & 11 \% \\ \text { Sulphonylurea(SU) alone } & 16 \% \\ \text { Metformin + SU } & 31 \% \\ \text { Insulin alone } & 29 \% \\ \text { Metformin+Insulin } & 11 \% \\ \text { SU+Insulin } & 02 \%\end{array}$

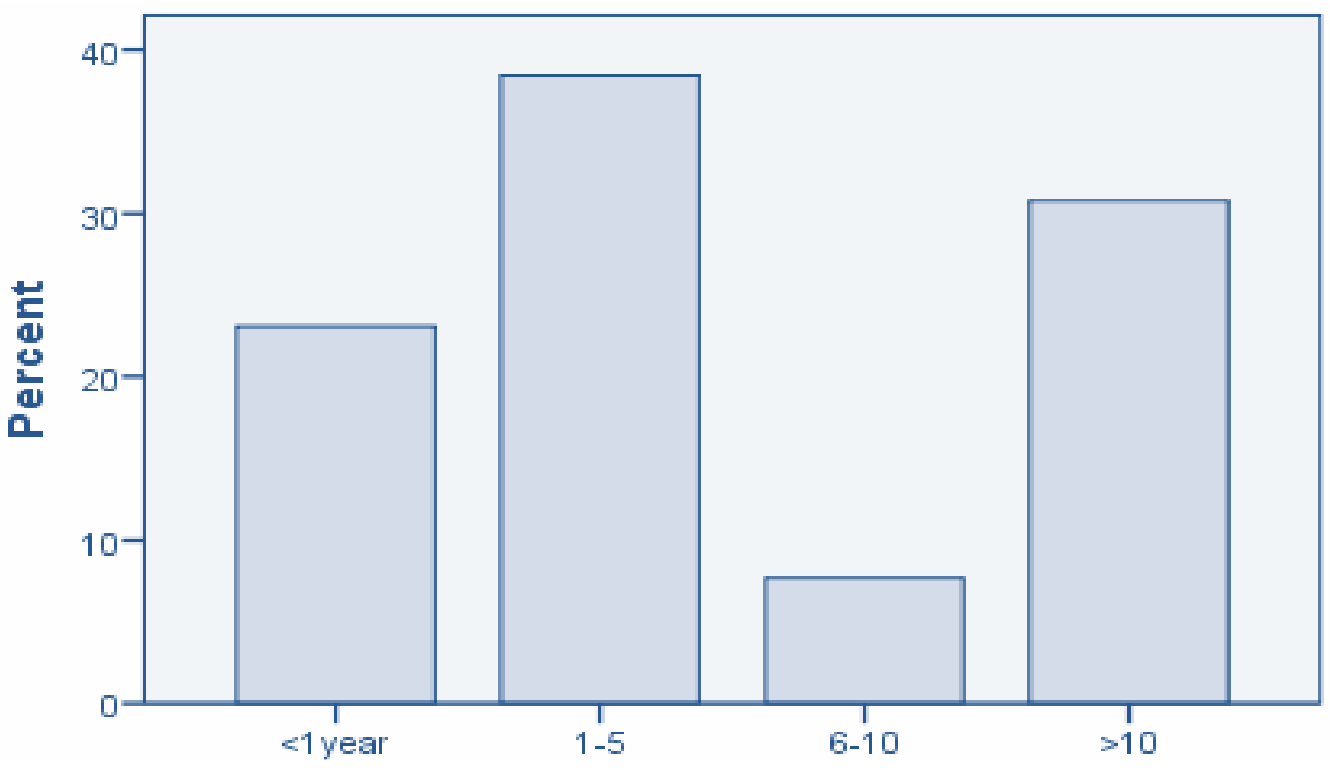

Figure 3: Duration of Insulin Use Among Study Participants 

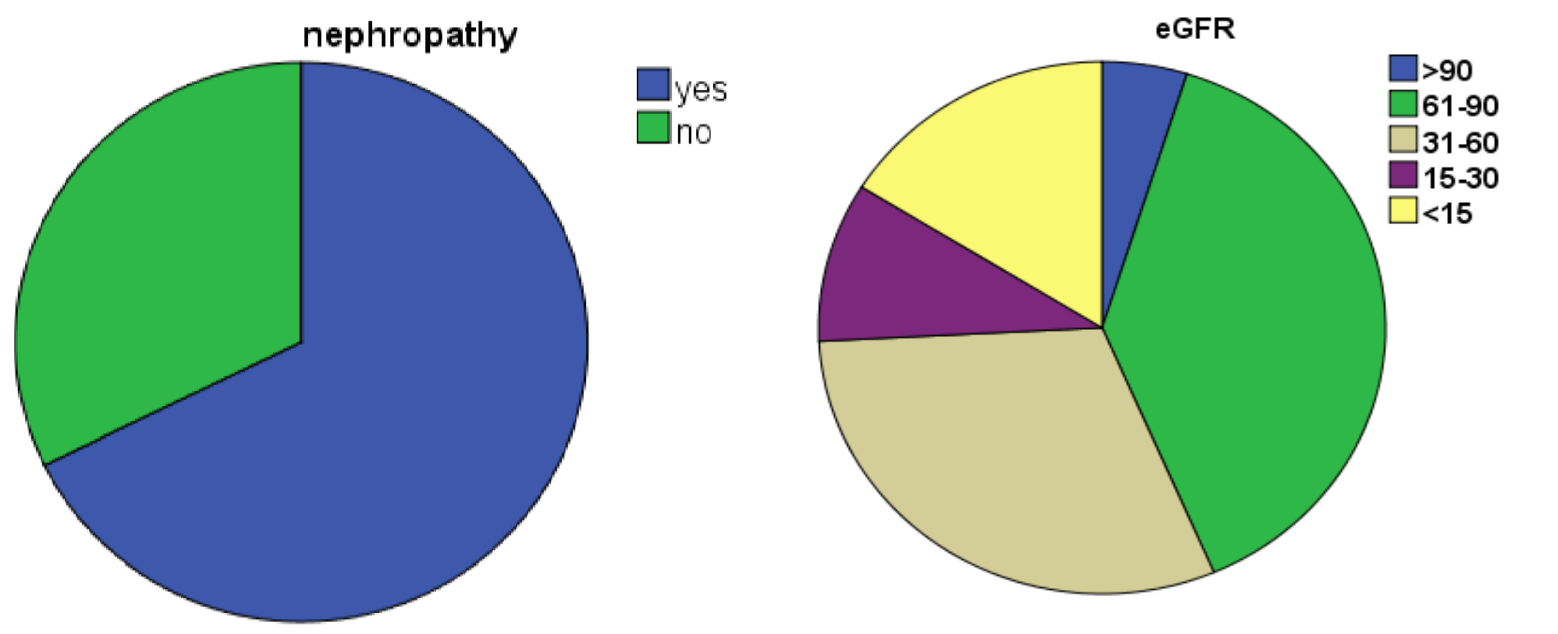

Figure 4: Prevalence of nephropathy and eGFR in study participants

\section{Discussion}

Hypoglycemia due to antidiabetic agents is a potentially serious complication among diabetic patients. It often jeopardizes the stringent glycemic control of diabetic patients in particular when optimum glycemic control is warranted.

There are well known traditional risk factors to develop hypoglycemia among diabetic patients such as worsening renal impairment, advancing age, complex drug regimes, insulin and sulphonylurea therapy. Our study once again reiterated the fact that the use of antidiabetic agents like insulin, sulphonylurea and renal impairment are the most common risk factors for hypoglycemic events in this study population.

Among the total study population about $48 \%$ (30 out of 62 ) were on sulphonylurea at the time of presentation and the most common sulphonylurea used was gliclazide. Nineteen percentage $(63 \%)$ of them were on a combination of sulphonylurea and metformin and another 10 was on sulphonylurea alone. Even though gliclazide said to be the safest drug in renal impairment, 7 patients on gliclazide monotherapy had hypoglycemia with estimated glomerular filtration rate less than $60 \mathrm{mg} / \mathrm{dl}$.

In contrast to the popular belief that metformin is a safer agent in terms of hypoglycemia, surprisingly 7 patients in this study experienced symptomatic hypoglycemia while on metformin monotherapy. And it is important to note that six of them had evidence of nephropathy and five patients had a subnormal eGFR of $<60 \mathrm{ml} / \mathrm{min}$. It reemphasizes the fact that metformin in the presence of nephropathy irrespective of the degree of renal impairment could result in symptomatic hypoglycemia.

Duration of diabetes per se did not have a significant impact on risk of developing hypoglycemia in contrast to the evidences published in the literature (4). Most of the patients in this study had diabetes for less than 10 years $(42 \%)$ followed by $35 \%$ who had it for $11-20$ years while $23 \%$ had it for more than 20 years. The reason could be explained by the fact that with the chronicity of the illness patients become more aware of warning symptoms and able to prevent a severe hypoglycemic episode. This attribution is further strengthened by the observation in this study that $2 / 3$ of the patients have presented with their first episode of hypoglycemia and the number of patients gradually decline with subsequent episodes.

Duration of insulin therapy did not have a significant impact on hypoglycemic events. Previous studies have shown that the duration of insulin therapy has a positive correlation with the risk of hypoglycemia however this study failed to show any correlation (5).

Interestingly, vast majority of the patients $(87 \%)$ in this study were aware of the fact that sugary drink should be taken immediately on detection of warning symptoms. But, despite the good knowledge, only $37 \%$ of patients have received first aid before reaching hospital. Reasons behind this gap between the knowledge and practice should be analyzed and addressed properly in order to prevent further events. Unfortunately, none of the patient in this study was aware of home Glucagon injection which is widely used by the patients in other part of world as an immediate remedy for a hypoglycemic event. 
A national online study done in United States of America among 2500 diabetic patients, also revealed the knowledge regarding the risks and causative factors of hypoglycemia was not optimal (10). An Indian study which again analyzed the knowledge of hypoglycemia among type 2 diabetic patients in a tertiary care hospital concluded that $66 \%$ of the study population had good knowledge on warning symptoms, precipitating factors and emergency management (11). But, only one third of patients were aware of complications of unattended hypoglycemia. In contrast to that finding, $77 \%$ of our patients $(48 / 62)$ were well aware of the fact that untreated or prolonged hypoglycemia could result in permanent brain damage or death. But, two third of the patients were not aware that warning symptoms can be absent or minimal in patients with long standing diabetes, meanwhile only $25 \%$ of study population had the knowledge that hypoglycemia per se could precipitate an acute coronary or cerebral event. Even though our patients were having fairly good knowledge on some of the complications they lack awareness on some important aspects.
Thus, patient education should be optimized, which requires a dedicated approach with repetitive advices and support. It is worth spending time with diabetic patients because experiencing a hypoglycemic episode is so panicking. Fear of such event will make the patients to become noncompliant to their treatment. This will, without doubt, lead to devastating complications of poor glycemic control. So, avoiding hypoglycemia is equally important to other pharmacological and non-pharmacological management of diabetes in order to prevent morbidity and mortality.

\section{Conclusion}

Hypoglycemia is still remaining a challenge for patients and clinicians who are involved in the management of diabetes. Choice of hypoglycemic agent should be individualized based on duration of diabetes and medical comorbidities.

\section{References}

1. American Diabetes Association. Standards of medical care in diabetes-2016. Diabetes Care. 2016;39(suppl 1):S1-S106.

2. UK Prospective Diabetes Study (UKPDS) Group. Intensive blood-glucose control with sulphonylureas or insulin compared with conventional treatment and risk of complications in patients with type 2 diabetes (UKPDS 33). Lancet. 1998;352:837-53.

3. Frequency of severe hypoglycemia requiring Emergency treatment in Type 1 and type 2 Diabetes. A population based study of health service resource use. Diabetes Care. 2003 Apr; 26(4): 1176-1180.

4. Henderson JN, Allen KV,Deary IJ, Frier BM. Hypoglycemia in insulin treated type 2 diabetes:frequency, symptoms and impaired awareness. Diabet Med. 2003;20:1016-1021.

5. ES Wijewickrama, UCL Hewage, DJS Fernando. Knowledge of hypoglycemia in patients with diabetes. Ceylon Med J. 2003 Jun;48(2):60.

6. Chelliah A, Burge MR. Hypoglycemia in elderly patients with diabetes mellitus : causes and strategies for prevention. Drugs Aging. 2004;21(8):511-30.

7. P Katulanda, DAV Rathnapala, R Sheriff, DR Matthews. Province and ethnic specific prevalence of Diabetes among Sri Lankan adults. Sri Lanka Journal of Diabetes Endocrinology and Metabolism. 2011;1: 2-7.

8. Katulanda P et al. Prevalence and projections of diabetes and pre-diabetes in adults in Sri Lanka-Sri Lanka Diabetes, cardiovascular study (SLDCS). Diabet Med. 2008 Sep;25(9):1062.

9. Van DalemJudith, Brouwers Martijn CGJ, Stehouwer Coen,DA, Krings André, Leufkens Hubert G M, Driessen Johanna H M et al. Risk of hypoglycaemia in users of sulphonylureas compared with metformin in relation to renal function and sulphonylurea metabolite group: population based cohort study. BMJ 2016; 354 :i3625.

10. Patients with type 2 Diabetes lack knowledge about hypoglycemia. American Association of Clinical Endocrinologists 20th Annual meeting and Clinical Congress. 2011;April 15.

11. Vanishree Shriraam, Shriraam Mahadevan, M.Anitharani, Nalini Sirala Jagadeesh, Sreelekh Bhaskara Kurup, T.A.Vidya and Krishna G. Seshadri.Knowledge of hypoglycemia and its associated factors among type 2 diabetes mellitus patients in a Tertiary Care Hospital in South India. Indian J EndocrinolMetab. 2015 May-Jun; 19(3): 378-382. 